\title{
Remarks on Entanglement, its Sources and Applications
}

\author{
M. ŻUKOWsKI
}

Instytut Fizyki Teoretycznej i Astrofizyki, Uniwersytet Gdański

Wita Stwosza 57, 80-952 Gdańsk, Poland

and

Institut für Experimentalphysik, Universität Innsbruck, 6020 Innsbruck, Austria

We shall discuss the central role played in the quantum information theory by entanglement. A generalized version of Bell's theorem will be presented, as well as methods in multiphoton interferometry to obtain correlations violating the Bell inequalities. Finally, we shall present some quantum informational applications of entanglement.

PACS numbers: 03.65.Ud, 03.67.-a, 42.50.Ar

\section{Introduction}

Current advances in the operational control of quantum phenomena enable us to use the paradoxical aspects of the quantum world as sources of practical applications. The main areas in which quantum phenomena can be harnessed to perform tasks impossible for classical theory of information are quantum cryptography (already a reliable technology), quantum communication (at the stage of birth, e.g. the teleportation experiments) and quantum computation (stunning theoretical results, breaking the limits of the Turing-type machines, but still far away from operational realizations).

The basic notion in the quantum information theory is that of the quantum bit or the qubit. Classical bits can take one of the two possible values 0 or 1 ("on" or "off", etc.). The qubits (the simplest quantum systems with pure states described by a two-dimensional Hilbert space, e.g. spins 1/2), since they are quantum entities, they can be in an arbitrary superposition of two basis states $|0\rangle$ and $|1\rangle$ :

$$
|q u b i t\rangle=\alpha|0\rangle+\beta|1\rangle \text {. }
$$


This superposition, and its generalizations to more complicated systems, is a source of quantum parallelism, which can be exploited to construct ultrafast quantum computer algorithms. Quantum algorithms are capable, in theory, of factorizing large numbers much faster than those based on the classical Turing principles. Within an entity as simple as qubit one finds the elementary quantum interference phenomena, one can also show that an unknown state of a qubit, or any other quantum system, cannot be cloned (which is the property that allows one to propose quantum cryptographic protocols).

If one has two qubits, the most bizarre pure state that they can be in is an entangled state

$$
\alpha|0\rangle_{1}|0\rangle_{2}+\beta|1\rangle_{1}|1\rangle_{2}
$$

with $\alpha, \beta \neq 0$. The state of Eq. (2) describes a coherent superposition of the two product states. No information whatsoever exists about which of the two possibilities is actually the case. This information can only be gained via an additional measurement, which destroys the superposition (just as any interaction with a third quantum system).

Another important property of the state (2) is that it does not make precise predictions about the results of an individual measurement on one of the two qubits. The qubits individually are in mixed states, e.g. the qubit 1 is described by the density matrix $\rho_{1}=|\alpha|^{2}|0\rangle_{11}\left\langle\left. 0|+| \beta\right|^{2} \mid 1\right\rangle_{11}\langle 1|$. If the pure state of the two qubits is maximally entangled, i.e. $|\alpha|=|\beta|$, all information is contained only in the joint properties described by (2), each individual qubit is in a completely random mixture of its possible states. As soon as measurement on one of the qubits is performed, the state of the other one is pure and well defined. This is independent of the spatial separation between the two qubits. Basically, all earlier studies of entanglement, starting with the famous Einstein-Podolsky-Rosen argument concentrated on this aspect of entangled states.

It turns out that entanglement is an essential ingredient in the quantum communication (e.g. the quantum teleportation protocol involves entanglement as a necessary resource) and quantum computation.

In this presentation we shall discuss the most paradoxical aspect of entanglement, namely the Bell theorem, as well as some optical methods of obtaining multiphoton entangled states. Finally, we shall briefly discuss quantum teleportation. First, its definition will be presented, then the experimental methods utilized in the first demonstration of teleportation of a polarization state of a photon, in which the photon in the original state to be teleported was emitted independently of the entangled pair employed in the quantum part of the teleportation channel.

\section{Bell theorem}

Before the advent of Bell theorem [1], despite Einstein's doubts [2], the question of the existence of a more detailed description of individual events in the mi- 
croworld, than the probabilistic one provided by quantum mechanics, was treated as interesting, however not falsifiable, and therefore as irrelevant as the question of "how many angels fit on the tip of the needle". In early sixties Bell proved [1] that the conflict between quantum mechanics and the realistic theories ${ }^{*}$ is confined to local ${ }^{\dagger}$ versions of such theories. His famous theorem is of profound scientific and philosophical consequences.

\subsection{Bell inequality}

Let us consider pairs of particles (say, photons) simultaneously emitted in well defined opposite directions. After some time the photons arrive at two very distant measuring devices A and B operated by two characters: Alice and Bob.

Their apparatuses have a knob which specifies, which dichotomic (i.e., two-valued, yes-no, 0-1, one bit) observable they actually measure..$^{\circ}$ One can assign to the two possible results the numbers +1 (for yes, bit value one) and -1 (for no, bit value nil)..$^{\S}$ Alice and Bob are at any time (also in a "delayed choice" mode, after an emission) free to choose the observables (knob settings) that they want to measure.

Let us assume that each photon pair carries full information (deterministic or probabilistic) on the values of the results of all possible experiments that can be performed on it (realism). Also, by locality, choices made by them which are simultaneous in certain reference frame cannot influence each other (in Alice's region of space-time, which contains the measurement event, there is no information whatsoever available on Bob's choice, and vice versa); the choice made on one side cannot influence the results on the other side.

For simplicity, let us assume that Alice chooses to measure either observable $\hat{A_{1}}$ or $\hat{A}_{2}$, and Bob either $\hat{B_{1}}$ or $\hat{B}_{2}$. Let us denote the hypothetical results that they may get for the $j$-th pair by $A_{1}^{j}$ and $A_{2}^{j}$, for Alice's two possible choices, and $B_{1}^{j}$ and $B_{2}^{j}$, for Bob's. The numerical values of these results $(+1$ or -1$)$ are defined by the two eigenvalues of the observables. Since, always either $\left|B_{1}^{j}-B_{2}^{j}\right|=2$ and $\left|B_{1}^{j}+B_{2}^{j}\right|=0$, or $\left|B_{1}^{j}-B_{2}^{j}\right|=0$ and $\left|B_{1}^{j}+B_{2}^{j}\right|=2$, with a similar property of Alice's hypothetical results the following relation holds:

$$
\left|A_{1}^{j} \pm A_{2}^{j}\right|\left|B_{1}^{j} \pm B_{2}^{j}\right|=0
$$

for all possible sign choices within (3) except one, for which one has 4 . Therefore

${ }^{*}$ Realism, the cornerstone of classical physics, is a view that any physical system (i.e. also a subsystem of a compound system) carries full information (deterministic or probabilistic) on results of all possible measurements that can be performed upon it.

${ }^{\dagger} \mathrm{A}$ theory is local if it assumes that information and influences cannot travel faster than light.

$\ddagger$ For example, for a device consisting of a polarizing beam splitter and two detectors behind its outputs, this knob would specify the orientation of the polarizer.

$\S$ We assume here perfect situation in which the detectors never fail to register a photon.

TNote that in the present discussion, only this idea openly goes beyond "what is speakable" in quantum mechanics. 
$\sum_{k=0,1 ; l=0,1}\left|\left[A_{1}^{j}+(-1)^{k} A_{2}^{j}\right]\left[B_{1}^{j}+(-1)^{l} B_{2}^{j}\right]\right|=4$.

Let us imagine now that $N$ pairs of photons are emitted, pair by pair ( $N$ is sufficiently large, $\sqrt{1 / N} \ll 1)$. The average value of the products of the local values for a joint test (often called the Bell correlation function), during which, for all photon pairs, a specific pair of observables, say $\hat{A}_{n}$ and $\hat{B}_{m}$, is chosen by the local observers, is given by

$$
E\left(A_{n}, B_{m}\right)=\frac{1}{N} \sum_{j=1}^{j=N} A_{n}^{j} B_{m}^{j}
$$

where $n=1,2$ and $m=1,2$. The relation (4) implies that for the four possible choices of pairs of observables the following "Bell" inequality [3-6] must be satisfiedll

$$
\begin{aligned}
& \sum_{k=0,1 ; l=0,1} \mid E\left(A_{1}, B_{1}\right)+(-1)^{l} E\left(A_{1}, B_{2}\right) \\
& \quad+(-1)^{k} E\left(A_{2}, B_{1}\right)+(-1)^{k+l} E\left(A_{2}, B_{2}\right) \mid \leq 4 .
\end{aligned}
$$

In the actual experiment only in part of the cases (say, approximately $1 / 4$-th) the given pair of observables would be measured, however if $N$ is very large, the correlation function obtained on a randomly pre-selected sub-ensemble** of emissions cannot differ too much from the one that would have been obtained for the full ensemble. ${ }^{\dagger \dagger}$ Therefore, for the values of the actually chosen measurements the inequality (6) also must hold.

To complete the Bell theorem one should find a quantum process which violates this inequality. ${ }^{\ddagger}$ Bell has shown such a violation for maximally entangled states (the first experiment is reported in [8]). In fact, predictions for any pure, non-factorizable (i.e. not necessarily maximally entangled) two-system state violate such inequality for some specific observables [9]. This is the case for a wide range of mixed states $[10,5]$. The inequality $(6)$ can be easily generalized to multiqubit systems, which are known to violate local realism even more strongly than pairs of qubits [11].

IThe famous Clauser-Horne-Shimony-Holt inequality [7] is implied by (6). However, the CHSH inequality, when generalized to an arbitrary number of qubits, does not lead to a set of inequalities which are necessary and sufficient for a local realistic description of the correlations. In contradistinction the present inequality does have this property.

** The sub-ensemble is selected by the choice of observables made by Alice and Bob before the actual measurements.

${ }^{\dagger \dagger}$ With $N \rightarrow \infty$ the difference must approach zero, otherwise we would suspect that these two magnitudes pertain to two different physical processes (i.e., a systematic error must be involved).

\#Violations of Bell inequalities imply that the conjunction of locality and realism does not hold for quantum systems. It is popular to say just that it is locality that cannot hold. However, such a claim has no grounds: quantum mechanics forbids instantaneous information transfer. 


\section{Optimal source of entangled systems: spontaneous parametric down conversion}

One can find in the literature very detailed theoretical descriptions of the parametric down conversion (PDC) process. Thus, we shall only give its essential traits and basic physics.

If one shines a strong linearly polarized monochromatic laser beam, or a quasi-monochromatic laser light pulse, on a suitably cut and oriented birefringent crystal endowed with a high quadratic nonlinearity, some pump photons spontaneously fission into pairs of photons of lower frequency (for historical reasons called signal and idler), Fig. 1. The process is quasi-elastic. Thus the frequencies of pump photon, $\omega$, signal, $\omega_{\mathrm{s}}$, and idler, $\omega_{\mathrm{i}}$, satisfy

$$
\omega \approx \omega_{\mathrm{s}}+\omega_{\mathrm{i}}
$$

(for the pulsed pump this relation still holds, however in this case the pulse frequency is not precisely defined). Since down conversion can occur anywhere within the laser illuminated part of the crystal, the geometry of the process leads to a constructive interference of the pair emissions into the so-called phase matched directions only. The photonic phase matched wave vectors satisfy (within the crystal):

$$
k_{\mathrm{p}} \approx k_{\mathrm{s}}+k_{\mathrm{i}}
$$

i.e., the emissions are strongly correlated directionally (again, for the pulsed case $\boldsymbol{k}_{\mathrm{p}}$ is not precisely defined). Due to the dependence of the speed of light on frequency, phase matching within a crystal cannot occur for all frequencies, and all emission directions, and thus into a given direction only specific frequencies are emitted. Knowing the frequency and the direction of an idler we can predict with a good accuracy corresponding parameters for the signal. The sharpness of Eq. (8) grows with the size of the crystal, and of the laser beam waist.

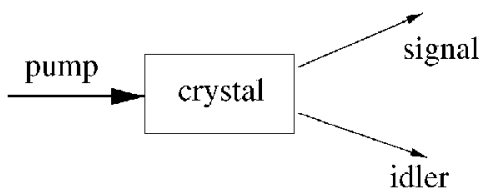

Fig. 1. Parametric down conversion.

Let us now discuss a more quantitative description of the PDC radiation. We shall consider a pulsed pump. We assume that: (i) the probability of a multiple emission from a single PDC is low; (ii) the laser pulse is not too short, i.e., the nonmonochromaticity of the pulse will not blur too much the strong angular correlation of the emissions (due to the effective energy and momentum conservation within the crystal). Thus, the photons can be still described as emitted in specified, very well defined directions. 
Under the approximations that: (i) only two phase matched directions are singled out (by a pair of pinholes in a diaphragm); (ii) the idler and signal frequencies satisfy perfect energy conservation conditions with the pump photons, which is described by a sharply peaked at the origin function $\Delta_{L}\left(\omega-\omega_{\mathrm{i}}-\omega_{\mathrm{s}}\right)$ which approaches Dirac's delta for $L \rightarrow \infty$ ( $L$ symbolically represents the crystal's size); (iii) the pump pulse is described as a classical wave packet (no-depletion) with one single direction for all wave vectors (the frequency profile of the pulse will be denoted by $g(\omega)$ ), the state of the photon pair emerging from the PDC source (plus the filtering system) via two phase matched beams $a$ and $d$ can be written as

$$
\left|\psi_{a d}\right\rangle=\int \mathrm{d} \omega_{1} \mathrm{~d} \omega_{2} \mathrm{~d} \omega \Delta_{L}\left(\omega-\omega_{1}-\omega_{2}\right) g(\omega) f_{a}\left(\omega_{1}\right) f_{d}\left(\omega_{2}\right)\left|\omega_{1} ; a\right\rangle\left|\omega_{2} ; d\right\rangle,
$$

where, e.g., the ket $|\omega ; e\rangle$ describes a single photon of frequency $\omega$ in the beam defined by the pinhole $e$, the function $g$ represents the spectral content of the pulse, $f_{e}$ is the transmission function of the filtering system in the beam $e$ (a pinhole and/or a filter).

If one removes trivial retardation effects, the amplitude to detect a photon at time $t_{x^{\prime}}$ by a detector monitoring the beam $x^{\prime}$ and another one at time $t_{y^{\prime}}$ by a counter in the beam $y^{\prime}$, provided the initial photon state was, say, $\left|\psi_{x y}\right\rangle$, can be written in a simple form as $A_{x y}\left(t_{x^{\prime}}, t_{y^{\prime}}\right)=\left(\left\langle t_{x^{\prime}} ; x^{\prime}\left|\left\langle t_{y^{\prime}} ; y^{\prime}\right|\right) \mid \psi_{x y}\right\rangle\right.$, where $|t ; b\rangle=\frac{1}{\sqrt{2 \pi}} \int \mathrm{d} \omega \mathrm{e}^{\mathrm{i} \omega t}|\omega ; b\rangle$. The elementary amplitudes of the detection process have a simple, intuitively appealing, form

$$
A_{x y}\left(t_{x}, t_{y}\right)=\frac{1}{\sqrt{2 \pi}} \int \mathrm{d} t G(t) F_{x}\left(t_{x}-t\right) F_{y}\left(t_{y}-t\right),
$$

where the functions denoted by capitals are the Fourier transforms: $H(t)=$ $\frac{1}{\sqrt{2 \pi}} \int \mathrm{d} \omega \mathrm{e}^{\mathrm{i} \omega t} h(\omega)$, for $h=f$ or $g$. Note that the time correlation between the detection of the idler and the corresponding signal photon is entirely determined by the band width of the detection system. For example this implies that in the limit of no filtering, when the functions $F(t)$ are approaching $\delta(t)$, the time correlation is extremely sharp, which can be illustrated by somewhat mathematically incorrect limiting case of $(10)$, namely $G\left(t_{x}\right) \delta\left(t_{x}-t_{y}\right)$ (in reality, one also has to take into account the phase matching function $\Delta$, and this imposes a sharp but finite time correlation for the PDC process). However, just a single filter will blur this correlation to around the inverse of the filter's band width, $\Delta T \approx 1 / \Delta \nu$ (the coherence time of the filtered radiation). The function $G(t)$ represents the temporal shape of the laser pulse and its presence in the formula simply indicates that (barring retardation) the signal and idler can be produced only when the pulse is present in the crystal.

If the birefringent crystal is cut in such a way that the so-called type-I phase matching condition is satisfied, both PDC photons are of the same polarization (if the pump beam is an ordinary wave, the down converted photons are extraordinary). Due to the phase matching condition (8) (single) photons of the same 
frequency are emitted into cones centered at the pump beam. By picking photons from a specially chosen cone one can have PDC radiation with both photons of equal frequency $\frac{1}{2} \omega_{\mathrm{p}}$. The selection can be done by a suitable pinhole arrangement in a diaphragm behind the crystal and/or with the use of filters. Pairs of pinholes can be pierced at points on a circle, drawn on the diaphragm, and centered about the pump beam. The pinholes of each pair should be bored at points symmetric with respect to the center of the circle. If a down-conversion photon passes through one of the pinholes, then the other photon will pass through the diametrically opposite pinhole. If there are two pairs of diametrically opposite pinholes, the state of the photon pair will be a superposition of passage through the two pairs of pinholes. The state describing the coherent superposition for the pair of photons to leave the aperture system with equal probability by either the pinholes $a$ and $d$ or $a^{\prime}$ and $c^{\prime}$ can be written as

$$
\left|\Psi^{A}\right\rangle=\frac{1}{\sqrt{2}}\left(\left|\psi_{a d}\right\rangle+\left|\psi_{a^{\prime} d^{\prime}}\right\rangle\right)
$$

For very sharp filtering this state can be practically described as $\frac{1}{\sqrt{2}}\left(|a\rangle|d\rangle+\left|a^{\prime}\right\rangle\left|d^{\prime}\right\rangle\right)$, where, e.g., $|a\rangle$ describes a particle going through the pinhole $a$, etc.

In the case of type-II phase-matching down conversion, idlers and signals are emitted into two cones. The pump has the extraordinary polarization and the two down-converted photons have orthogonal polarizations. Idler and signal from each pair have momenta of opposite transverse components (with respect to the momentum of the pump photons). Therefore, they are emitted into opposite sides of the pump beam. One can arrange the alignment in such a way that the two cones intersect along two lines. If additionally the frequencies associated with each of the cones are identical, there is no information whatsoever on the polarization of the individual photons travelling along the lines of intersection of the cones. Nevertheless, a joint property of the two photons is well defined: they have two opposite orthogonal polarizations, i.e. their polarizations are in the entangled state

$$
\frac{1}{\sqrt{2}}\left(|H\rangle|V\rangle+\mathrm{e}^{\mathrm{i} \phi}|V\rangle|H\rangle\right)
$$

where $H$ and $V$ denote opposite linear polarizations, and $\phi$ is determined by the properties of the crystal.

In summary, the down conversion process is an excellent source of pairs of photons entangled in frequencies, momenta (directions), and polarizations. The complementary aspect of the frequency entanglement are very sharp temporal correlations. In a standard Bell-type experiment these temporal correlations are used to identify detections associated with a single pair of emitted photons. The correlation of polarizations or directions of the photons give rise to data which reveal violations of Bell inequalities. 


\section{Entangling independently emitted particles}

All the progress in observation and handling of the two-particle entanglement still leaves some fascinating questions:

- Can one perform multi-particle higher order interference experiments for particles originating from independent sources (rather than for intense fields - e.g. interference experiments involving two superposed laser beams, or attenuated coherent fields)?*

- There is an old question posed already by Bell: can one have an "event ready detectors" test of Bell's inequalities? ${ }^{\dagger}$

- Is the phenomenon of entanglement confined only to particles originating from one source, or at least interacting with each other at a certain stage?

- Can one entangle particles that share no common past and are spatially separated? And if yes, then how strong can be such entanglement (is it strong enough to violate some Bell inequality)?

- Is it technologically feasible to transfer a pure state of one system to another one, which is independently emitted and spatially separated (quantum teleportation)?

- Is it possible to swap or teleport entanglement?

Yurke and Stoler [12] have suggested that entanglement may arise in the coincidence count rates of particles originating from independent sources. Such a scheme requires monitoring of the emissions with time resolution significantly sharper than the coherence time of the radiation fed into the interferometric setup [13]. These principles are of a general nature, and one must take them always into account when designing setups involving manipulations of states of independent quantum systems (e.g. teleportation, entanglement swapping, interactions in the prospective quantum computing gates), since they affect the quality of the processes.

\subsection{Generic situation}

Let us consider Fig. 2 which depicts a generic situation.

Two independent sources emit one pair of, for example, beam entangled particles each. A simplified representation of the resulting four-particle state is the product of two beam entangled states $\sqrt{1 / 2}\left(|a\rangle|b\rangle+\left|a^{\prime}\right\rangle\left|b^{\prime}\right\rangle\right)$ with $\sqrt{1 / 2}(|c\rangle|d\rangle+$ $\left|c^{\prime}\right\rangle\left|d^{\prime}\right\rangle$ ). Let us suppose that we register a particle in detector $i_{1}$ in coincidence with a particle in detector $i_{2}$ (the particles detected will be called idlers, the

*The earlier experiments involving interference of light originating from independent sources involved states (essentially the coherent ones) for which the particle interpretation is doubtful even at very low intensity.

${ }^{\dagger}$ Essentially, in such experiment the ensemble upon which the measurements are to be performed is defined before the actual measurements. 


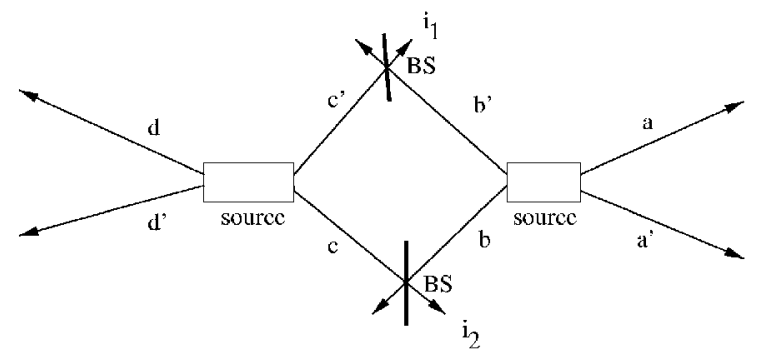

Fig. 2. Interference of two independent sources of entangled qubits. Entanglement swapping. BS stands for beam splitter.

other two particles will be called signals). Then, the state of the other particles collapses into the entangled state $\sqrt{1 / 2}\left(|a\rangle\left|d^{\prime}\right\rangle+\left|a^{\prime}\right\rangle|d\rangle\right)$. This is possible only provided the procedure of emission and selection of the four particles is such that one cannot ever know, not even in principle, which source produces the events at the particle detectors. This emerging entanglement is a consequence of both the initial entanglements from each source and the fact that coincident registration in detectors $i_{1}$ and $i_{2}$ projects the signal particles into the "Bell" state $2^{-1 / 2}\left(|b\rangle\left|c^{\prime}\right\rangle+\right.$ $\left.\left|b^{\prime}\right\rangle|c\rangle\right)$. The experiment can be arranged such that all registration events occur outside each other's light cones.

\subsection{Indistinguishability of the sources of particles}

To emit beam-entangled pairs, each source, e.g. parametric down conversion, must initially contain a parent particle whose momentum and energy is definite to some extent (see the section on beam entanglement). The emerging pairs of daughter particles will, in general, carry correlations in energy, momentum (the rationale for beam entanglement), spin (polarization), energy (and thus time). Any of these can be exploited to identify the true sibling and hence the source of the particle detected in $i_{1}$ or $i_{2}$, and thereby prevent entanglement of signals from forming. However, one can never exploit these correlations to reveal the origin of the particles provided:

1. for polarization (spin, etc.) correlations: if either all particles simply carry the same polarization, or in case of the initial polarization entanglement, if we place before the particle detectors a polarizer that selects just one polarization, chosen in such a way that the original polarization correlation is erased,

2. the energy correlations of true pairs (emitted by the same source) are operationally indistinguishable from mixed pairs (one particle from one source),

3. the temporal correlations of true pairs and of mixed pairs are operationally indistinguishable. 
Below we present several versions of operational conditions to achieve the last two goals, illustrated by the case of down converted photons.

\subsection{Coincident detection}

Even for ideal devices with perfect time resolution one always has to impose a finite time gate to define two counts as coincident. In the case of initial perfect temporal correlation, if one of the entangled particles passes through a frequency filter, the band width $\Delta \nu_{\mathrm{f}}$ of the filter implies that the detection time of one particle now is determined by the registration time of its partner particle up to around its coherence time $T_{c} \approx 1 / \Delta \nu_{\mathrm{f}}$ and vice versa (see the previous section). Thus in the setup under consideration we consider filtering only in the beams leading to the $i$ detectors.

\subsubsection{Path distinguishability}

Let us consider as coincident two particles (idlers) arriving at the detectors $i_{1}$ and $i_{2}$ within a time window $\tau_{\mathrm{i}}=T_{\mathrm{c}}$. This implies that two other particles (signals) are within the setup. Let us assume that some detectors will sooner or later register both signals. Now, a signal caught earlier can be thought with a higher probability to be paired with the idler registered earlier. Therefore, the larger both the time separation between the registrations of the particles and that between the registrations of the signals, the more signal path information we have. The resulting partial distinguishability of the paths taken by the signals leads to a reduction of the two particle interference contrast in a possible two-particle interference process involving the signals.

\subsubsection{Ultracoincidence criterion}

To overcome these problems, one can impose subcoherence time coincidence on the registration of the idlers [13]:

$$
\Delta T_{\text {coinc }} / T_{\mathrm{c}} \ll 1 \text {. }
$$

This implies high visibility in an experiment involving the signals: the idlers are registered within such a narrow time window that we have no signal path information (since path information resolution in time correlations is $T_{\mathrm{c}}$ ). However, due to current technical limitations (the time resolution of optical detectors $\tau \approx 0.5 \mathrm{~ns}$ $\gg T_{\mathrm{c}} \approx 1 \mathrm{ps}$ ) this method is currently unrealizable.

Alternatively, let us suppose that the sources are of a pulsed nature, with the duration of the pulse $\Delta T_{\mathrm{p}}$ (and synchronized pulses), spectral width $\sigma_{\mathrm{p}} \approx 1 / \Delta T_{\mathrm{p}}$, and that the band widths of the filters of the idler radiation are narrower than $\sigma_{\mathrm{p}}$, i.e.

$$
\Delta T_{\mathrm{p}} / T_{\mathrm{c}} \ll 1
$$

Therefore the unfiltered particles (signals) must appear within a coincidence window defined by the pulse duration; i.e., one has $\left|t_{A}-t_{B}\right| \leq \Delta T_{\mathrm{p}}$. But the filtered 
particles (here, idlers) due to the action of the filters lose their original tight time correlation with their partner signals (which is after the filtering of the order of $T_{\mathrm{c}}$ ). The origin of the detection events at the idler detectors is lost, and the signals may reveal high visibility two-particle interference. Their joint state is close to the maximally entangled one.

This method is perfectly feasible, and has been tested in several experiments in which new multiphoton states were generated, or quantum informational processes were demonstrated [14-16]. Below is an example.

\section{Exemplary quantum communication process: quantum teleportation}

Quantum teleportation [17] is the operational protocol, Fig. 3, which enables one to transfer the quantum state of one system, say 1 , to another quantum system, 3. The transfer can be obtained by performing a joint ("Bell-state") measurement on 1 and a third system 2, originally EPR entangled with 3, and then unitarily transforming 3 according to the outcome of this measurement. Teleportation separates the complete information in 1 into two parts: a classical part carried by the outcome 3 of the joint measurement on 1 and 2 , and a nonclassical part carried by the prior entanglement between 2 and 3 .

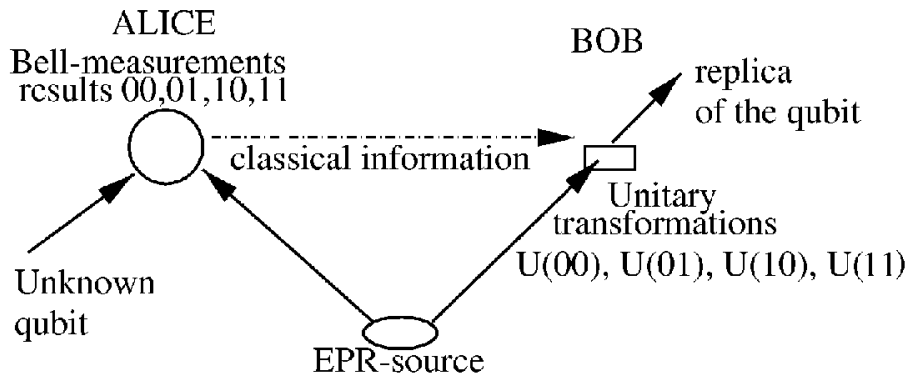

Fig. 3. The principle of quantum teleportation. Alice receives a qubit in a state which is unknown to her and to Bob. She is also in a possession of one of the two maximally entangled qubits (the other one is in Bob's lab). Alice performs a joint measurement on her two qubits in the Bell-state basis. She receives, with equal probability, one of the possible results, totally unrelated with the state of the qubit. Next she transmits a two-bit message to Bob, via classical means, informing him about her result. Depending on the message Bob performs one of the four unitary transformations on his qubit from the entangled EPR pair (two qubits are called EPR entangled, if they are in a maximally entangled state). As a result, his qubit emerges in a state which is a replica of the, now nonexistent, unknown state of left hand side qubit. 
Note that this process is possible despite the fact that one cannot clone quantum objects. That is, having a single quantum object in certain unknown arbitrary state $|\psi\rangle$ there is no physical process that leads to two quantum systems, each in the state $|\psi\rangle$. Note that this implies that there is no way to measure the state in which a quantum system is. Had this been possible one could clone by first measuring the state of the initial system, and then preparing two, three, or more systems in this state.

The formal description of the teleportation process in the simplest case of qubits runs as follows. The initial three-particle state is

$$
\left(a|x\rangle_{1}+b|y\rangle_{1}\right) \sqrt{\frac{1}{2}}\left(|x\rangle_{2}|y\rangle_{3}-|y\rangle_{2}|x\rangle_{3}\right),
$$

where $x, y$ denote two orthogonal states of the three subsystems. The complex parameters $a$ and $b$ are determined by the state preparation procedure of Cecil, and are unknown to both Alice and Bob. A different decomposition of the state above is given by

$$
\begin{aligned}
& \frac{1}{2}\left[\left|\psi^{-}\right\rangle_{12}\left(-a|x\rangle_{3}-b|y\rangle_{3}\right)+\left|\psi^{+}\right\rangle_{12}\left(-a|x\rangle_{3}+b|y\rangle_{3}\right)\right. \\
& \left.\quad+\left|\phi^{+}\right\rangle_{12}\left(b|x\rangle_{3}+a|y\rangle_{3}\right)+\left|\phi^{-}\right\rangle_{12}\left(-b|x\rangle_{3}+a|y\rangle_{3}\right)\right]
\end{aligned}
$$

where the four Bell states were introduced

$$
\begin{aligned}
& \sqrt{\frac{1}{2}}\left(|x\rangle_{1}|y\rangle_{2}-|y\rangle_{1}|x\rangle_{2}\right)=\left|\psi^{-}\right\rangle_{12}, \\
& \sqrt{\frac{1}{2}}\left(|x\rangle_{1}|y\rangle_{2}+|y\rangle_{1}|x\rangle_{2}\right)=\left|\psi^{+}\right\rangle_{12}, \\
& \sqrt{\frac{1}{2}}\left(|x\rangle_{1}|x\rangle_{2}-|y\rangle_{1}|y\rangle_{2}\right)=\left|\phi^{-}\right\rangle_{12}, \\
& \sqrt{\frac{1}{2}}\left(|x\rangle_{1}|x\rangle_{2}+|y\rangle_{1}|y\rangle_{2}\right)=\left|\phi^{+}\right\rangle_{12} .
\end{aligned}
$$

Alice performs Bell state measurements on the particles 1 and 2. With equal probability she gets one of the four possible results. Alice's measurement projects the particle 3 on one of the four states, which are spelled out in Eq. (18). The consequence of the collapse of the full wave vector due to Alice's Bell-state measurement is that Bob's qubit is in a specific state. Performing one of four unitary transformations depending on Alice's specific result, Bob can transform his particle into the original qubit. This is why he must know the result of Alice's measurement, which has to be transmitted to him via a classical communication channel. These four unitary transformations are independent of the state of particle 1, and are related solely to the result of Alice's Bell-state measurement. If Alice's measurement gives $\left|\psi^{-}\right\rangle$, she sends to Bob a message, say 00, and he simply does nothing (i.e. performs an identity transformation). This is due to the fact that after such 
a result at Alice's side the state of particle 3 is already a copy of the original state of 1 (the overall minus sign is of course irrelevant). If she gets $\left|\psi^{+}\right\rangle$, and sends 01 , he performs a unitary transformation which leaves the $x$ state intact, and changes the phase of the $y$ by $\pi$. If her result is $\left|\psi^{-}\right\rangle$, and the message is 10, Bob's transformation must be the one that interchanges the states $x$ and $y$. And finally, if the message is 11 , which means that the result was $\left|\psi^{+}\right\rangle$, he performs both aforementioned transformations one after another. In all cases the transformed state of 3 is a perfect replica of the initial state of 1 . In the process the original qubit disappears during the Bell-state measurement, and thus Bob's qubit is not a copy but a teleported reappearance of the original.

Alice's Bell-state measurement does not convey any information about the original state. It is important to stress that this procedure can be used both to teleport pure states and mixed ones.

\subsection{Bell-state measurement}

The major problem in the experimental verification of quantum teleportation is the Bell-state measurement of two independently created quantum systems. This means that these two qubits have to be measured in such a way that it is not possible to infer which detection event refers to which source. Thus the methods discussed in the previous section have to be employed. The experimental problem is that thus far no complete Bell-state analyzer exists in the laboratory for any kind of quantum systems. This is due to the fact that complete Bell-state analysis requires nonlinear interaction between the two qubits, which has not been realized so far.

Nevertheless, exploiting the fact that only $\left|\psi^{-}\right\rangle$is antisymmetric, while other Bell states are symmetric, partial Bell-state analysis is possible for two qubits which are in this case polarization states of two independent photons ${ }^{\ddagger}$, i.e. $x$ and $y$ in the previous formulas stand now for linear polarization directions. This can be done with the use of a 50-50 polarization neutral beam splitter. It is very easy to show that if photons 1 and 2 , in the polarization Bell state $\left|\psi^{-}\right\rangle$, hit the beam splitter at two opposite entrance ports, the interference at the beam splitter causes them to exit the beam splitter in such a way that one has one photon in each output ports. In the case of the three other polarization Bell states both photon exit via one port.

All that sounds very simple, but sole application of a beam splitter cannot do the trick if the photons, as it should be in a teleportation protocol, are created independently, i.e. they originate from two independent sources. To this end one has to additionally use all the methods to make independent photons indistinguishable which were presented in the previous section. Only then the required interference effect occurs. In the first demonstration of the full teleportation

$\ddagger$ Photons are bosons. Their full state is symmetric. Only the polarization degrees of freedom are antisymmetric in the discussed case. 
process [14] the EPR pair and the qubit to be teleported were provided by two independent emissions of a down converter.

\section{D. Back to the foundations of quantum physics: teleportation and the interpretation of the collapse postulate}

The quantum teleportation protocol directly utilizes the collapse postulate of the standard quantum theory of measurement. The controversy around this postulate is usually associated with its seemingly instantaneous, nonlocal character. Quantum teleportation sheds a new light onto this problem. Let us assume that Alice knows the pure state that she wants to teleport to Bob. Then, immediately after her Bell-state measurement she knows exactly the pure state on Bob's side. Nevertheless, until the classical, sub-luminal transmission of her result reaches Bob there is absolutely no way he can utilize the purity of his state. For him the state remains maximally mixed.

Nevertheless, if he performs measurements on an ensemble of his particles, and after that obtains the information from Alice which members of his ensemble were associated with which of Alice's results, then the ensemble can be split into four parts, each revealing effects characteristic of the one of the four pure states, into which the particle 3 collapses after Alice's measurements [18].

The moral of the story is that the collapse of the wave vector at Bob's side occurs instantaneously for Alice (as she is immediately in possession of the information on her local result). Whereas, for Bob, the collapse has practical value only after Alice gave him the information - no super-luminal utilization of the collapse is possible. The local state of the system is described in accordance with locally accessible information.

This work is supported by the State Committee for Scientific Research grant No. 5 P03B 08820.

\section{References}

[1] J.S. Bell, Physics 1, 195 (1964).

[2] A. Einstein, B. Podolsky, N. Rosen, Phys. Rev. 47, 777 (1935).

[3] H. Weinfurter, M. Żukowski, Phys. Rev. A 64, 010102(R) (2001).

[4] R.F. Werner, M.M. Wolf, preprint quant-ph/0102024 at (http:/xxx.lanl.gov), 2001.

[5] M. Żukowski, C. Brukner, preprint quant-ph/0102039 at (http:/xxx.lanl.gov), 2001.

[6] C. Brukner, M. Żukowski, A. Zeilinger, preprint quant-ph/0106119 at (http:/xxx.lanl.gov), 2001.

[7] J. Clauser, M. Horne, A. Shimony, R. Holt, Phys. Rev. Lett. 23, 880 (1969).

[8] S.J. Freedman, J.S. Clauser, Phys. Rev. Lett. 28, 938 (1972).

[9] N. Gisin, Phys. Lett. A 154, 201 (1991). 
[10] R. Horodecki, P. Horodecki, M. Horodecki, Phys. Lett. A 200, 340 (1995).

[11] D.M. Greenberger, M. Horne, A. Zeilinger, in: Bell's Theorem, Quantum Theory, and Conceptions of the Universe, Ed. M. Kafatos, Kluwer Academic, Dordrecht 1989, p. 73.

[12] B. Yurke, D. Stoler, Phys. Rev. A 46, 2229 (1992).

[13] M. Żukowski, A. Zeilinger, M.A. Horne, A.K. Ekert, Phys. Rev. Lett. 71, 4287 (1993); M. Żukowski, A. Zeilinger, H. Weinfurter, Ann. NY Acad. Sci. 755, 91 (1995).

[14] D. Bouwmeester, J.W. Pan, K. Mattle, M. Eibl, H. Weinfurter, A. Zeilinger, Nature 390, 575 (1997).

[15] J.-W. Pan, D. Bouwmeester, H. Weinfurter, A. Zeilinger, Phys. Rev. Lett. 80, 3891 (1998).

[16] D. Bouwmeester, K. Mattle, J.-W. Pan, H. Weinfurter, A. Zeilinger, M. Żukowski, Appl. Phys. B 67, 749 (1998).

[17] C.H. Bennett, G. Brassard, C. Crepeau, D. Jozsa, A. Peres, W.K. Wootters, Phys. Rev. Lett. 70, 1895 (1993).

[18] A. Zeilinger, Philos. Trans. R. Soc. Lond. 1733, 2401 (1997). 